\title{
Nondestructive measurements of residual stress in air plasma-sprayed thermal barrier coatings
}

\author{
Peng Jiang ${ }^{1}$, Liuyu Yang ${ }^{1}$, Yongle Sun ${ }^{2}$, Dingjun $\mathrm{Li}^{1,3}$, Tiejun Wang ${ }^{1, *}$
}

${ }^{1}$ State Key Laboratory for Strength and Vibration of Mechanical Structures, Department of Engineering Mechanics, Xi'an Jiaotong University, Xi'an, China

${ }^{2}$ Welding Engineering and Laser Processing Centre, School of Aerospace, Transport and Manufacturing, Cranfield University, Cranfield, MK43 0AL, United Kingdom

${ }^{3}$ State Key Laboratory of Long-Life High Temperature Materials, Dongfang Steam Turbine Co., Ltd., Deyang, China.

\section{Graphical abstract}

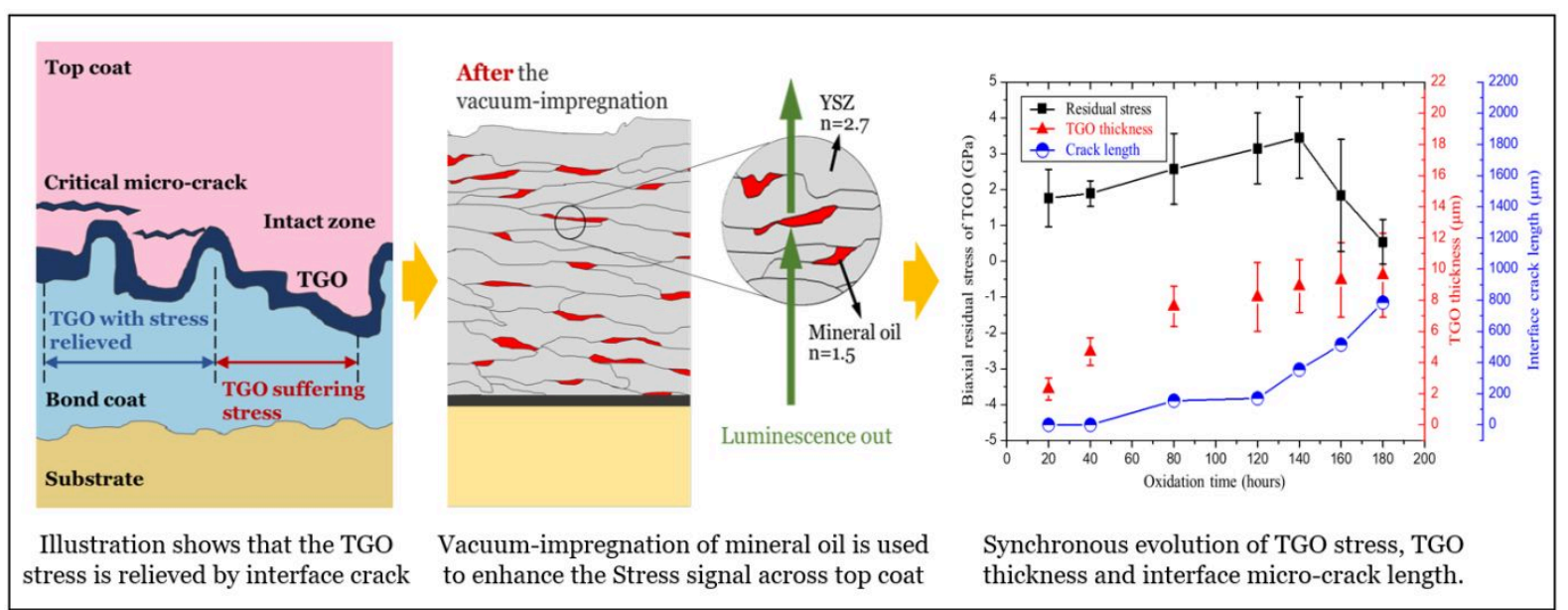

\begin{abstract}
Premature spallation of thermal barrier coatings (TBCs) is a critical issue during the service of gas turbines, and nondestructive evaluation is crucial to address this problem.
\end{abstract}

Herein, a novel approach that indicates delamination by measuring the residual stress 
evolution of thermally grown oxide (TGO) for air plasma spraying (APS) TBCs is proposed and verified via the combination of photoluminescence piezo-spectroscopy (PLPS) and X-ray computed tomography. A mineral-oil-impregnating approach and a cold-mount low-shrinkage epoxy-mounting approach are used to alleviate the signal attenuation by pores and micro-cracks in APS TBCs, improving the detectable PLPS signal and X-ray transmission for stress measurement and delamination characterization, respectively. We have nondestructively measured the TGO residual stress mapping in APS TBCs and its evolution with oxidation. Furthermore, the evolution of TGO morphology and critical micro-cracks are obtained by X-ray computed tomography. The synchronous evolution of TGO residual stress, TGO thickness, and critical micro-cracks as a function of oxidation time is obtained and correlated. The transition point, as experimentally identified, at which the TGO stress starts to drop, agrees well with the critical moment of micro-crack coalescence. This directly verifies that the TBC delamination can be effectively indicated by residual stress evolution of TGO in APS TBCs.

Keywords: Thermal barrier coating, Debonding, Residual stress, Photoluminescence piezo-spectroscopy, X-ray computed tomography

\section{INTRODUCTION}

There is a demand for more efficient and powerful gas turbines, which are characterized by higher operating temperatures, longer lifetimes and other features ${ }^{1-3}$. 
This demand has led to great challenges in the development of advanced thermal protection technologies, among which thermal barrier coatings (TBCs) are regarded as one of those most promising to meet the demand ${ }^{4-7}$. Typically, TBCs consist of the following four layers ${ }^{5,}$ : (i) the superalloy substrate, which is the main load-bearing constituent; (ii) the ceramic top coat (TC), which is usually composed of $6-8$ wt.\% yttria-stabilized zirconia (YSZ) and acts as a temperature insulator; (iii) an aluminum-containing bond coat $(\mathrm{BC})$ between the substrate and the $\mathrm{TC}$, which is usually composed of MCrAlY (where $\mathrm{M}$ is $\mathrm{Ni}$ and/or $\mathrm{Co}$ ) and used for alleviating the thermal expansion mismatch stress between the aforementioned two layers; and (iv) a thermally grown oxide (TGO), which forms between $\mathrm{TC}$ and $\mathrm{BC}$ when they are exposed to high temperature and provides oxidation resistance. Each of these constituent layers presents marked differences in physical, thermal, and mechanical properties, and all contribute to determine performance and durability. Typically, the TBCs are fabricated using either electron-beam physical vapor deposition (EB-PVD) or air plasma spraying (APS $)^{9}$. The APS technique is of relatively low cost and usually adopted in gas turbine blades of energy generators. For APS-based TBCs, the TC is fabricated by spraying the powders layer by layer and features a lamellar microstructure, resulting in randomly distributed micro-cracks and micro-pores, which lead to a low thermal conductivity.

The premature spallation of TBCs is a critical issue during the service of gas turbines, and the fundamental failure mechanisms have been mostly revealed ${ }^{10-18}$. Nondestructive detection of delamination plays an important role in warning of failure and guiding mitigation, but it still faces many technical chanlleges ${ }^{19}$, especially for APS 
TBCs on gas-turbine blades. A reliable detection of the damage at the $\mathrm{TC} / \mathrm{BC}$ interface can not only facilitate structural health monitoring, but also provide a more rational recoating interval for a condition-based maintenance strategy, which enables reduction in TBC maintenance cost. Several testing techniques have been developed for detection of delamination in TBCs, e.g., ultrasound, THz waves, active thermography, eddy current, electrochemical impedance, and reflectance-enhanced luminescence. The limitations of these techniques in the applications to TBCs are summarized as follows. Neither ultrasonic waves nor $\mathrm{THz}$ waves are able to transmit throughout the YSZ topcoat because of the large signal attenuation caused by the porous microstructure ${ }^{20-24}$. In the active thermography method, the temperature difference between the delaminated and non-delaminated areas in TBCs is usually not evident for accurate detection ${ }^{25-27}$. The signal of TGO thickness detected by the eddy current testing method depends on the micro-constituents in TGO, such as $\mathrm{Al}_{2} \mathrm{O}_{3}, \mathrm{NiO}$, and $\mathrm{Cr}_{2} \mathrm{O}_{3}$, which complicate the interpretation of the acquired signal for reliable detection. The electrochemical impedance can be used to reveal the degradation of TBCs; however, the impedance signal is found to be sensitive to test parameters such as electrode size, voltage amplitude, and environment temperature ${ }^{28-30}$. In the reflectance-enhanced luminescence method, a rare-earth-based luminescent sublayer is embedded immediately above the TC/BC interface, and it can produce sufficient luminescence intensity to identify the delaminated regions. However, this method suffers from the occurrence of false detections caused by dirty spots and smears on the surface $31-33$.

Instead of direct detection of delamination, the interfacial stress relaxation due to 
delamination can be used as an indicator of delamination and thus the nondestructive measurement of stress state at the interface is not only useful for stress analysis but also promising as an effective indirect approach for delamination detection ${ }^{34-39}$. It is well known that TGO is subjected to high compressive residual stresses when the TC/TGO interface is intact (Fig. 1). However, after long-time high temperature exposure, TGO thickness increases and consequently delamination occurs and grows with time, accompanied by a significant decrease in the TGO residual stress (Fig. 1). The coupling of stress relaxation and delamination implies that TGO stress measurement can provide rich information for structural integrity assessment. Equally importantly, nondestructive measurement of TGO stress is less difficult than nondestructive detection of delamination.

The photoluminescence piezo-spectroscopy (PLPS) method is recognized as a reliable and practical technique for nondestructive measurement of the stress in TGO and it can be further used for indirect detection of delamination. The PLPS method is based on the correlation between $\mathrm{Cr}^{3+}$ luminescence spectrum peak shifts and the stress states. Ma and Clarke ${ }^{40}$ first presented the relationships between the measured fluorescence shifts and the stress states in single-crystal and polycrystalline ceramics. Subsequently, Christensen et al. ${ }^{34}$ determined the residual stress in TGO of EB-PVD TBCs after different oxidation times through measuring the fluorescence shift of $\mathrm{Cr}^{3+}$ ion within the TGO. They found that the TGO stresses fall in the range of 3.5-4 GPa. Using PLPS method, Heeg et al. ${ }^{36}$ experimentally identified the TGO behavior under thermal cycling throughout its lifetime towards eventual failure. Fukuchi et al. ${ }^{38}$ and 
Manero et al. ${ }^{39}$ focused on the high-resolution spatial mapping of the stress in TGO, which provides a better understanding of the mechanisms leading to failure. However, most of them are not applicable to APS TBCs. This is because the ceramic TC has a large quantity of pores and horizontal micro-cracks (Fig. 2a), which strongly attenuate the fluorescence signal. Furthermore, the TGO in APS TBCs exhibit more irregular and complex morphologies than that in EB-PVD TBCs; thus, the delamination in APS TBCs is more complicated. For APS TBCs, other ions, such as $\mathrm{Eu}^{3+}$, was introduced into the ceramic TC as a sublayer-stress sensor ${ }^{41}$. However, the correlations between TGO morphology, residual stress, critical micro-cracks near interface and resultant delamination have not been revealed thus far, significantly limiting the further application of PLPS method to APS TBCs.

In the present work, delamination in APS TBCs is indicated by measuring the TGO residual stress. The residual stress and delamination are correlated with TGO morphology through the combination of PLPS and in-situ X-ray computed tomography (CT) methods. A mineral oil impregnating approach and a cold-mount low-shrinkage epoxy mounting approach are adopted to alleviate signal attenuation due to pores and micro-cracks in APS TBCs, and these approaches enable reliable detection of the PLPS signal and X-ray transmission, respectively. We have first obtained the residual stress mapping of TGO in APS TBCs. Furthermore, the TGO residual stress, TGO thickness, and delamination, as a function of oxidation time, are characterized synchronously, along with analyses and discussion on the experimental results. 


\section{EXPERIMENTAL}

\subsection{Sample preparation and high-temperature oxidation}

The substrate of the studied TBCs was made of nickel-based superalloy Hastelloy $X$ with dimensions of $3 \mathrm{~mm} \times 3 \mathrm{~mm} \times 3 \mathrm{~mm}$ and $2 \mathrm{~mm} \times 1 \mathrm{~mm} \times 1 \mathrm{~mm}$ for the PLPS testing and the X-ray CT testing, respectively. The BC was fabricated using commercial

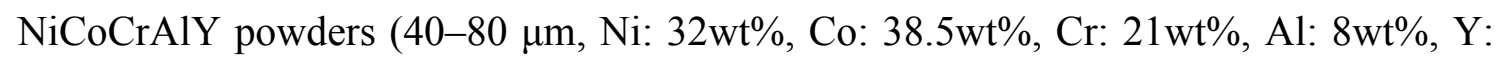
$0.5 \mathrm{wt} \%$, Sulzer-Metco) and the high-velocity oxygen fuel (HOVF) spraying method. The TC was fabricated using commercial $8 \mathrm{wt} \%$ YSZ powders $(45-100 \mu \mathrm{m}$, purity 99.99\%, Institute of Process Engineering, Chinese Academy of Science) and the APS method. The thicknesses of the TC and $\mathrm{BC}$ were $300 \mu \mathrm{m}$ and $150 \mu \mathrm{m}$, respectively. Detailed parameters used in the thermal spraying can be found in our previous study ${ }^{42}$.

The TBC specimens were heated in a laboratory muffle furnace for $180 \mathrm{~h}$ at 1150 ${ }^{\circ} \mathrm{C}$ from the as-sprayed state to spallation. At different exposure times (i.e., 20, 40, 80, $120,140,160$, and $180 \mathrm{~h}$ ) the specimens were temporarily removed from the furnace, cooled to room temperature, and then tested. Following the completion of each test, the specimens were returned to the furnace for the next heat treatment period.

\subsection{Nondestructive measurement of residual stress in TGO beneath porous YSZ TC}

The PLPS technique is used to measure residual stress in TGO, which examines the spectral luminescence of TGO via laser excitation. As porosity and micro-cracks are inherent in APS TBCs, the luminescence signal is attenuated when light travels from the dense YSZ (refractive index is 2.7) to air (refractive index is 1) present in the pores, as shown in Fig. 2(a). To reduce the mismatch in the refractive indices, the APS TBCs 
samples were vacuum-impregnated with mineral oil (Johnson \& Johnson, New Brunswick, NJ) before the PLPS testing, as shown in Fig. 2(c). The mineral oil is a highly refractive material with a refractive index of 1.5 . The impregnation was conducted at five different vacuum degrees, i.e., $10^{-4}, 10^{-2}, 10^{0}, 10^{2}$, and $10^{4}$, to determine the optimum vacuum condition. The pump-down time, vacuum holding time, and pump-up time were set as $30 \mathrm{~min}, 60 \mathrm{~min}$, and $30 \mathrm{~min}$, respectively.

The PLPS data were collected by Horiba HR800 spectroscopy equipment (Horiba, Kyoto, Japan). A $532 \mathrm{~nm}$ laser with $50 \mathrm{~mW}$ of power was used to excite a luminescence response from $\mathrm{Cr}^{3+}$ ions in TGO. The acquisition time was set as $20 \mathrm{~s}$ at $100 \%$ laser power. The spectral resolution was set as $0.3 \mathrm{~cm}^{-1}$ with a $1800 \mathrm{~g} / \mathrm{mm}$ grating. The objective magnitude was selected to be $10 \times$ to ensure a laser beam spot size much larger than the grain size of $\mathrm{TGO}^{41}$. The laser was focused on the TBCs sample surface, and the previous study ${ }^{39}$ confirmed an insignificant influence of the depth of focus. An in-plane area of $3 \mathrm{~mm} \times 3 \mathrm{~mm}$ was measured point-by-point by moving the focused laser beam to generate a residual stress map. Forty rows were scanned with a spatial resolution of $75 \mu \mathrm{m}$ in both the vertical and horizontal scanning directions.

For data processing, a Lorentz curve algorithm was employed to fit the spectrum and determine the peak position. The peak is shifted to smaller wavenumbers upon application of compressive stress. The peak shifts can be determined through comparing the peak position for a stressed TGO with the reference peak position for a stress-free TGO. Then, the relationship between the peak shift and compressive stress was used to calculate the TGO residual stress, which is expressed in following equation ${ }^{41}$ : 


$$
v_{s}-v_{0}=\Pi_{i j} \times \sigma_{i j},
$$

where $v_{S}$ is the peak position for the stressed state, $v_{0}$ is the peak position for the stress-free state, $\Pi_{i j}$ is piezo-spectroscopic coefficient (PSC), and $\sigma_{i j}$ is the stress state in the crystallographic basis of the host crystal. As the TGO can be regarded as a thin film, the stress state is presumed to be biaxial (i.e., $\overline{\sigma_{X X}}=\overline{\sigma_{Y Y}}, \overline{\sigma_{Z Z}}=0$ ), and the biaxial residual stress $\overline{\sigma_{X X}}$ or $\overline{\sigma_{Y Y}}$ can be finally determined as ${ }^{41}$

$$
v_{s}-v_{0}=\frac{2}{3}\left(\Pi_{11}+\Pi_{22}+\Pi_{33}\right) \times \sigma_{x x}
$$

Herein, PSC values of $7.59 \mathrm{~cm}^{-1} / \mathrm{GPa}$ and $7.61 \mathrm{~cm}^{-1} / \mathrm{GPa}$ for $\mathrm{R} 1$ and $\mathrm{R} 2$, respectively, were used according to Manero et al. ${ }^{39}$.

\section{$2.3 X$-ray tomography characterization of TBCs}

Microscopic characterization of TBCs is usually based on cross-section scanning. This means only a two-dimensional microstructure is captured, which may not be representative for the actual microstructure. Here, the three-dimensional microstructure of the coating was nondestructively imaged by X-ray CT using a Sanying Voxel-3000 Nano X-ray microscope (Sanying, China), as schematically shown in Fig. 3. When the $\mathrm{X}$-ray is passed through the TBCs specimen, the different layers, i.e., the TC, BC, TGO, substrate, as well as the air (in pores), can be distinguished by the dissimilar X-ray attenuation coefficients and then rendered in images of different grayscale levels. Owing to the high attenuation coefficient of the porous YSZ material and nickel-based superalloy, the TBCs sample was embedded in a cold mounting resin to enhance signal. The substrate was also slightly thinned down to $1 \mathrm{~mm}$, during which the cooling water was applied to avoid overheating the substrate. 
The X-ray tube was operated at $225 \mathrm{kV}$ with a beam current of $0.1 \mathrm{~mA}$. A lead glass X-ray filter was used to improve the polychromatic X-ray beam for the test. The optimal value of transmission is presumed to be $23 \%-35 \%$, which would result in the elimination of the noise in the images. A $10 \times$ objective lens was used to achieve a pixel size of less than $0.75 \mu \mathrm{m}$ with a field of view of almost $2 \times 2 \mathrm{~mm}^{2}$. The tomography scans were performed with 4000 projections at an angular increment of $0.045^{\circ}$. The exposure time was selected as 6.0 s per angular increment. A $2048 \times 2048$ CCD pixel detector was used to record the projections. This characterization technique allowed the observation of the microscopic features at the same location for a single TBCs sample as a function of oxidation time and temperature.

The projections were then reconstructed to reveal the three-dimensional TBCs microstructure, with particular attention placed on the TGO morphology and interface delamination. The reconstruction was conducted using nano Voxel CT-Pro software (Sanying, China). The image analysis, including filtering, segmentation and quantification, was performed using Avizo software (FEI, version 9). It should also be mentioned that a top-hat segmentation method was used here, which is believed to have great advantage in extracting small elements from given images.

It is important to note that, the mineral-oil-impregnation and cold-mount low-shrinkage epoxy-mounting would not affect the residual stress state in TBCs. The mineral oil used in PLPS test was a low-viscosity liquid and impregnated by capillary force in porous YSZ ceramic. The capillary force was very slight and, thus, could be ignored when compared with the residual stress in the TGO (1-5 GPa). The cold-mount 
epoxy was used for mounting samples. This is helpful in holding the microstructure and stress state in TBCs. In addition, after each PLPS and X-ray CT test, the samples were heated in a laboratory muffle furnace at $1150{ }^{\circ} \mathrm{C}$ for the next heat treatment period. Thus, both the mineral oil and cold-mount epoxy disappeared and would not induce any additional stress.

\section{RESULTS AND DISCUSSIONS}

Fig. 4(a) presents a comparison of $\mathrm{Cr}^{3+}$ luminescence intensities, which are nondestructively detected from the TGO at different vacuum degrees, i.e., $10^{-4}, 10^{-2}, 10^{0}$, $10^{2}$, and $10^{4} \mathrm{~Pa}$. Solid lines represent results after the mineral-oil impregnation, and dotted lines represent results from the untreated samples. For each vacuum degree, five points are collected (points A, B, C, D, and E on impregnated sample, and points F, G, $\mathrm{H}$, I, and $\mathrm{J}$ on the sample without impregnation). As might be expected, the luminescence intensities have been dramatically enhanced after the treatment for all vacuum degrees, demonstrating the effectiveness of the approach we proposed. The maximum intensities of $\mathrm{Cr}^{3+}$ luminescence for each vacuum degree are also described in Fig. 4(b). It is noted that, the luminescence intensity sharply decreases when the vacuum decreases from $10^{-4}$ to $10^{0}$, whereas it tends to be insensitive when the vacuum is lower. In particular, there does appear to be a horizontal asymptote representing a critical value of the vacuum degree, of approximately $10^{2}$, below which the luminescence intensity is not significantly impacted by the vacuum strength. According to the results, a vacuum degree of $10^{-4}$ was selected for the subsequent determination of 
residual stress in TGO.

The distributions of biaxial residual stresses in TGO in APS TBCs as a function of oxidation time have been nondestructively measured, as shown in Fig. 5. Each map represents 1600 measurement points in total over the entire sample. All maps have been generated using the R1 spectral peaks, which are higher than the R2 peaks, resulting in less influence of bimodal stress on the fitting procedures. The average thermal oxidation life of TBCs was determined to be approximately $180 \mathrm{~h}$. The residual stress is color-coded in Figure 5, and the stress-scale is adjusted to optimize the color contrast. Clearly, the residual stress is overwhelmingly compressive. The TGO stress was approximately $1 \mathrm{GPa}$ after the first $20 \mathrm{~h}$ of oxidation (Fig. 5a); then, it increased to 2 GPa after $40 \mathrm{~h}$ of oxidation (Fig. 5b) and to $3 \mathrm{GPa}$ after $80 \mathrm{~h}$ of oxidation (Fig. 5c). It continued increasing to 4-5 GPa after $140 \mathrm{~h}$ of oxidation (Fig. 5e). At approximately $88.9 \%$ of TBCs life (after 160 h oxidation), the residual stress of TGO dropped abruptly and exhibits a significantly non-uniform distribution (Fig. 5f). Low-stress and high-stress areas indicate micro-cracks and intact zone, respectively. Hence, it can be deduced that critical micro-cracks initiated at different locations after long-time oxidation. Following the initiation of micro-cracks, the residual stresses further decreased to approximately $0-1 \mathrm{GPa}$, until the spallation of the entire TBCs at the $180 \mathrm{~h}$ oxidation lifetime (Fig. 5g).It should be also noted that the non-homogeneity of the stress distribution gradually reduced during the oxide growth, implying that the final spallation was caused by the coalescence of these critical micro-cracks.

To synchronously capture the evolution of TGO and critical micro-cracks during 
the high-temperature oxidation, the X-ray CT test was conducted. Fig. 6(a) shows the 3D color-rendered 3D microstructure image of the APS TBCs after $20 \mathrm{~h}$ oxidation. The nickel-based superalloy substrate is marked in green at the bottom, in which the orange points represents high-Z material. The NiCoCrAlY bond coat in the middle layer is labeled in blue, and the YSZ top coat is in yellow. The TGO at TC/BC interface is marked in black. Pores and cracks in the TC and at the interface are marked in dark blue. Magnifications of the microstructures are presented in Figs. 6(b)-6(d). From these images the YSZ TC, TGO, BC, and superalloy substrate can be reliably distinguished. This enables the observation of TGO growth and microcrack evolution at a specific location of the same TBC sample, which can be correlated with the TGO residual stress and confirm the effectiveness of the delamination indicated by reduction in TGO residual stress.

To reveal the detailed microstructural features for a specific location, we examined the CT slices and tracked the microstructural evolution of a locally enriched TGO section. These sequential CT slices enable us to observe the TGO growth and identify the initiation of the critical micro-cracks. In such a way, the evolution of TGO morphology, critical micro-cracks, and TC pores are captured as a function of thermal oxidation time, with a series of internal microstructure images taken from the $3 \mathrm{D}$ X-ray tomography results at the same location in the same TBC sample, as shown in Fig.7. The grey contrast in the images originates from the different X-ray absorption coefficients of each layer. The TC and substrate are in lighter gray, whereas the BC appears darker. The pores and micro-cracks that are distributed in the TC and TGO at 
the interface are in black. The TGO layer can be clearly observed above the BC layer after $20 \mathrm{~h}$ oxidation (Fig. 7a); and the TGO growth became faster in the following $120 \mathrm{~h}$ oxidation (Fig. 7d). The TC/BC interface remained intact before $40 \mathrm{~h}$ oxidation (Fig. 7b), although many defects existed near the interface. The critical micro-cracks gradually initiated after $80 \mathrm{~h}$ oxidation, which are located right above the TGO up to approximately 10-20 $\mu \mathrm{m}$ (Figs. 7c and 7d). This cracking behavior is different from that observed in the EBPVD TBCs, in which the micro-cracks are mainly formed at the TC/TGO interface ${ }^{4}$. After $120 \mathrm{~h}$ oxidation, the rate of TGO growth becomes slow, and the TGO thickness remained approximately constant (Figs. 7e and 7f). In the meanwhile, the critical micro-cracks that nucleated at different locations converged, leading to the final spallation of the YSZ TC (Fig. 7f). Notably, the variation in TGO thickness and micro-cracks is consistent with the evolution of the TGO residual stress. In particular, the residual stress dropped drastically (Fig. 5g) when the coalescence of these micro-cracks occurred, which is in accordance with previous numerical results ${ }^{[43,}{ }^{44]}$. This confirms the effectiveness of the approach using TGO residual stress reduction as an indicator of delamination for APS TBCs.

The quantification of critical micro-cracks was performed by measuring the lengths of the micro-crack, as well as the TGO thickness, using the images obtained from the CT slices for through-thickness sections. It should be mentioned that 10 measurements of TGO thickness were carried out at random locations for each oxidation time, thereby capturing the statistics of the thickness of the TGO exhibiting microstructural inhomogeneity. Furthermore, the synchronous evolution of TGO residual stress, TGO 
thickness, and critical micro-crack length are obtained for different oxidation times, as shown in Fig. 8. Evidently, the TGO thickness increased rapidly to $8.2 \mu \mathrm{m}$ after the first $120 \mathrm{~h}$ oxidation and then became stable with an average value of $9.6 \mu \mathrm{m}$, following a sub-parabolic growth law. The crack length increased slowly at the initial stage but then increased rapidly to approximately $800 \mu \mathrm{m}$ over the rest of the oxidation time. The entire evolution of the TGO residual stress can be divided into two stages, i.e., the stress development stage and the stress relaxation stage. In the stress development stage (0-140 h oxidation, $0 \%-77.8 \%$ of TBC life), the stress accumulated owing to the TGO growth. The stress first rose rapidly to a mean value of approximately $1.76 \mathrm{GPa}$ after 20 h oxidation (11.1\% of TBC life). Then, the stress increased gradually until reaching a peak value of approximately $3.45 \mathrm{GPa}$ after $140 \mathrm{~h}$ oxidation $(77.8 \%$ of TBC life). The peak stress of TGO can be defined as a spallation stress. In the stress relaxation stage (160-180 h oxidation, $77.8 \%-100 \%$ of TBC life), the stress dropped rapidly from the peak and then decreased at a more or less constant rate, implying that most micro-cracks merged quickly at a critical moment. The drop in the TGO residual stress is mainly due to the coalescence of the micro-cracks near the interface, as demonstrated by the evolution curves of the micro-crack length (blue lines in Fig. 8). The standard deviation of the TGO residual stress in the relaxation stage is larger than that in the stress development stage. This can be mainly attributed to the high heterogeneity of TGO in APS TBCs. In addition, the turning point at which the TGO stress started to fall is in good correlation with the coalescence moment of the micro-cracks. This provides a clear verification that TBCs delamination can be effectively indicated by measuring the 
residual stress in TGO of APS TBCs.

\section{CONCLUSION}

A novel PLPS method is used to nondestructively measure the residual stress in TGO of APS TBCs subjected to high temperature oxidation. X-ray CT was employed to characterize the microstructural evolution during TGO growth. The residual stress has been correlated with oxidation and delamination, providing insights into the different aspects of the failure process. The conclusions are as follows:

(1) Mineral-oil-impregnation and cold-mount low-shrinkage epoxy-mounting methods were adopted to alleviate the PLPS and X-ray signal attenuation by pores and micro-cracks in APS TBCs, thereby enabling reliable measurement of residual stress and characterization of microstructural evolution, respectively. The vacuum degree was found to have great effect on the luminescence transmissivity through YSZ ceramic coatings. In particular, there is a critical vacuum value of approximately $10^{2}$, below which the luminescence intensity is not significantly impacted.

(2) For the first time, the residual stress distribution in the TGO of APS TBCs, as well as its evolution with oxidation time, was nondestructively measured. The stress evolution process can be divided into two stages, i.e., the development stage and the relaxation stage. In the development stage, the stress accumulated to a peak value of 3.45 GPa owing to the growth of TGO. In the relaxation stage, the stress dropped rapidly after the onset of peak stress but decreased at a constant rate afterward.

(3) The TGO thickness followed a sub-parabolic growth law with a final thickness of $9.6 \mu \mathrm{m}$. The critical micro-cracks were initiated after $80 \mathrm{~h}$ oxidation and were mainly 
located right above the TGO up to approximately 10-20 $\mu \mathrm{m}$ in height. The crack length increased slowly at the initial stage and then accelerated over the rest of the oxidation time.

(4) The residual stress, TGO thickness, and critical micro-cracks are different factors responsible for the final failure and their synchronous evolution as a function of oxidation time has been obtained. The residual stress increased with TGO growth until micro-crack nucleation. The transition point at which the stress in TGO started to fall is in good correlation with the critical moment of the micro-crack coalescence. This observation provides a direct verification that the $\mathrm{TBC}$ delamination can be effectively indicated by measuring the residual stress in TGO of APS TBCs.

(5) In the future, we will develop a portable TGO detection system, based on the proposed method, applicable to the preparatory test for topcoat delamination inspection, which allows flexibility in matching actual turbine blade and coatings with curved surfaces.

\section{ACKNOWLEDGMENTS}

This work was supported by the National Natural Science Foundation of China (11902240), China Postdoctoral Science Foundation funded project (221055), the fund of State Key Laboratory of Long-life High Temperature Materials, and the fund of Innovative Scientific program of CNNC. 


\section{REFERENCES}

1. D.L. Poerschke, R.W. Jackson, C.G. Levi, Silicate deposit degradation of engineered coatings in gas turbines: progress toward models and materials solutions, Annu. Rev. Mater. Res. 47 (2017) 297-300.

2. N.P. Padture, Advanced structural ceramics in aerospace propulsion, Nat. Mater. 15 (2016) 804-809.

3. E. Campo, V. Lupinc, High temperature structural materials for gas turbines, Metall. Sci. Technol. (2013).

4. N.P. Padture, Thermal barrier coatings for gas-turbine engine applications, Science 296 (2002) 280-284.

5. W. Fan, Y. Bai, Review of suspension and solution precursor plasma sprayed thermal barrier coatings, Ceram. Int. 42 (2016) 14299-14312.

6. V. Kumar, K. Balasubramanian, Progress update on failure mechanisms of advanced thermal barrier coatings: A review, Prog. Org. Coat. 90 (2016) 54-82.

7. A.G. Evans, D.R. Mumm, J.W. Hutchinson, G.H. Meier, F.S Pettit, Mechanisms controlling the durability of thermal barrier coatings, Prog. Mater. Sci. 46 (2001) 505-553.

8. R.A. Miller, Thermal barrier coatings for aircraft engines: history and directions, J. Therm. Spray Technol. 6 (1997) 35-42.

9. T.J. Wang, X.L. Fan, Y.L. Sun, L.C. Su, Y. Song, B.W. Lv, The stresses and cracks in thermal barrier coating system: A review, Acta Mech. Solida Sin. 37 (2016) 477-517. 
10. T. Sadowski, P. Golewski. Loadings in thermal barrier coatings of jet engine turbine blades, (2016).

11. I.T. Spitsberg, D.R. Mumm, A.G. Evans, On the failure mechanisms of thermal barrier coatings with diffusion aluminide bond coatings, Mater. Sci. Eng. A 394 (2005) 176-191.

12. K.W. Schlichting, N.P. Padture, E.H. Jordan, M. Gell, Failure modes in plasma-sprayed thermal barrier coatings, Mater. Sci. Eng. A 342 (2003) 120-130.

13. E.P. Busso, L. Wright, H.E. Evans, L.N. McCartney, S.R.J. Saunders, S. Osgerby, J. Nunn, A physics-based life prediction methodology for thermal barrier coating systems, Acta Mater. 55 (2017) 1491-1503.

14. B. Li, X.L. Fan, H. Okada, T.J. Wang, Mechanisms governing the failure modes of dense vertically cracked thermal barrier coatings, Eng. Fract. Mech. 189 (2018) 451-480.

15. B.W. Lv, X.L. Fan, D.J. Li, T.J. Wang, Towards enhanced sintering resistance: Air-plasma-sprayed thermal barrier coating system with porosity gradient, J. Eur. Ceram. Soc. 38 (2018) 1946-1956.

16. G.R. Li, L.S. Wang, W.W. Zhang, G.J. Yang, X.F. Chen, W.X. Zhang, Tailoring degradation-resistant thermal barrier coatings based on the orientation of spontaneously formed pores: From retardation to self-improvement, Compos. Pt. B-Eng. 181 (2020) 1359-8368.

17. M.J. Liu, M. Zhang, Q. Zhang, G.J. Yang, C.X. Li, C.J. Li, Evaporation of Droplets in Plasma Spray-Physical Vapor Deposition based on energy compensation 
between self-cooling and plasma heat transfer, J. Therm. Spray Technol. 26 (2017) 1641-1650.

18. Q.L. Li, P. Song, Q. Dong, C. Hua, C.M. Deng, L. Li, B.J. Zheng, J.S. Lu, Effect of partial crystallization of an amorphous layer on the mechanical properties of ceramic/metal-glass coating by thermal spraying, Ceram. Int. $45 \quad$ (2019) 18803-18813.

19. T. Fukuchi, T. Ozeki, M. Okada, T. Fujii, Nondestructive inspection of thermal barrier coating of gas turbine high temperature components, IEEJ Trans. Electr. Electron. Eng. 11 (2016) 391-400.

20. N. Fuse, T. Fukuchi, Nondestructive measurement of coated steels and thermal barrier coating using terahertz waves, IEEJ Trans. Electr. Electron. Eng. 134 (2014) $745-748$.

21. T. Fukuchi, N. Fuse, M. Okada, T. Ozeki, T. Fujii, M. Mizuno, K. Fukunaga, Topcoat thickness measurement of thermal barrier coating of gas turbine blade using terahertz wave, Electr. Eng. Jpn. 189 (2014) 1-8.

22. Y. Zhao, J. Chen, Z. Zhang, Nondestructive characterization of thermal barrier coating by noncontact laser ultrasonic technique, Opt. Eng. 54 (2015) 094104.

23. Y. Zhao, J. Chen, Z. Zhang, Laser ultrasonic evaluation of bonding layer in thermal barrier coating, Proc. SPIE (2015) 9438.

24. Y. Zhao, J.W. Chen, Z.Z. Zhang, Y.N. Zhu, Nondestructive evaluation of residual stress and TGO by using laser ultrasonic method, 2015, in: SPAWDA 2015. IEEE, 2015 p.411-417. 
25. C.W. Bu, Q.J. Tang, Y.L. Liu, F.Y. Yu, C. Mei, Y.W. Zhao, Quantitative detection of thermal barrier coating thickness based on simulated annealing algorithm using pulsed infrared thermography technology, Appl. Therm. Eng. 99 (2016) 751-755.

26. M. Schweda, T. Beck, J. Malzbender, L. Singheiser, Damage evolution of a thermal barrier coating system with 3-dimensional periodic interface roughness: Effects of roughness depth, substrate creep strength and pre-oxidation, Surf. Coat. Technol. 276 (2015) 368-373.

27. M. Schweda, T. Beck, M. Offermann, L. Singheiser, Thermographic analysis and modelling of the delamination crack growth in a thermal barrier coating on Fecralloy substrate, Surf. Coat. Technol. 217 (2013) 124-128.

28. D.X. Chen, C.B. Wan, W.G. Tian, M.C Pan, Calibration of micro-capacitance measurement system for thermal barrier coating testing, Rev. Sci. Instrum. 89 (2018) 064703.

29. L. Yang, W. Zhu, C.F. Li, Y.C. Zhou, N.G, Wang, Y.G. Wei, Error and modification in thermal barrier coatings measurement using impedance spectroscopy, Ceram. Int. 43 (2017) 4976-4983.

30. W.L. Chen, M. Liu, J.F. Zhang, Impedance Analysis of 7YSZ Thermal barrier coatings during high-temperature oxidation, J. Therm. Spray Technol. 25 (2016) 1596-1603.

31. J.I. Eldridge, T.J. Bencic, C.M. Spuckler, J. Singh, D.E. Wolfe, Delamination-indicating thermal barrier coatings using YSZ: Eu sublayers, J. Am. Ceram. Soc. 89 (2006) 3246-3251. 
32. J.I. Eldridge, T.J. Bencic, Monitoring delamination of plasma-sprayed thermal barrier coatings by reflectance-enhanced luminescence, Surf. Coat. Technol. 201 (2006) 3926-3930.

33. C.L. Li, X.L. Fan, P. Jiang, X.C. Jin, Delamination-indicating of atmosphere-plasma-sprayed thermal barrier coating system using $\mathrm{Eu}^{3+}$ luminescence mapping, Mater. Lett. 222 (2018) 41-44.

34. R.J. Christensen, D.M. Lipkin, D.R. Clarke, K. Murphy, Nondestructive evaluation of the oxidation stresses through thermal barrier coatings using $\mathrm{Cr}^{3+}$ piezospectroscopy, Appl. Phys. Lett.,69 (1996) 3754.

35. J.A. Nychka, D.R. Clarke, Damage quantification in TBCs by photo-stimulated luminescence spectroscopy, Surf. Coat. Technol. 146-147 (2001) 110-116.

36. B. Heeg, V.K. Tolpygo, D.R. Clarke, Damage evolution in thermal barrier coatings with thermal cycling, J. Am. Ceram. Soc. 94 (2011) s112-s119.

37. I. Hanhan, E. Durnberg, G. Freihofer, P. Akin, S. Raghavan, Portable Piezospectroscopy system: non-contact in-situ stress sensing through high resolution photo-luminescent mapping, J. Instrum. 9(2014) P11005-P11005.

38. T. Fukuchi, S. Eto, M. Okada, T. Fujii, Evaluation of applicability of compact excitation light source to detection of thermally grown oxide layer in thermal barrier coating for gas turbines, Electr. Eng. Jpn. 190 (2015) 1-8.

39. A. Manero, A. Selimov, Q. Fouliard, K. Knipe, J. Wischek, C. Meid, A.M. Karlsson, M. Bartsch, S. Raghavan, Piezospectroscopic evaluation and damage identification for thermal barrier coatings subjected to simulated engine 
environments, Surf. Coat. Technol. 323 (2017) 30-38.

40. Q. Ma, D.R. Clarke, Stress measurement in single-crystal and polycrystalline ceramics using their optical fluorescence, J. Am. Ceram. Soc. 76 (1993) 1433-1440.

41. P. Jiang, X.L. Fan, Y.L. Sun, H.T. Wang, L.C. Su, T.J. Wang, Thermal-cycle dependent residual stress within the crack-susceptible zone in thermal barrier coating system, J. Am. Ceram. Soc. 101 (2018) 4256-4261.

42. P. Jiang, X.L. Fan, Y.L. Sun, D.J. Li, B. Li, T.J. Wang, Competition mechanism of interfacial cracks in thermal barrier coating system, Mater. Des. 132 (2017) $559-566$.

43. B. Li, X.L. Fan, K. Zhou, et al., Effect of oxide growth on the stress development in double-ceramic-layer thermal barrier coatings, J. Ceram. Int. 43 (2017) $14763-14774$.

44. B. Li, X.L. Fan, T.J. Wang, et al., Interfacial fracture behavior of double-ceramic-layer thermal barrier coating system with segmented structure, J. Eng. Fract. Mech. 201 (2018) 13-28. 


\section{Figure captions}

Figure 1. Schematics of the intact zone at which TGO suffers residual stress and the cracked interface at which TGO stress is relieved.

Figure 2. Schematics showing (a) coating with pores (b) luminescence attenuated by porous ceramic coating. (c) Luminescence penetrating the ceramic coating vacuum impregnated with mineral oil.

Figure 3. Schematic describing the X-ray computed tomography test on the TBC sample, which was embedded in a cold mounting resin.

Figure 4. Fig. 4 (a) Comparison of the detected intensities of $\mathrm{Cr}^{3+}$ luminescence emitted by the TGO and passing through the YSZ TC, before and after the mineral-oil-impregnation treatment, at a vacuum degree of $10^{-4}$. (b) Change in average intensities of $\mathrm{Cr}^{3+}$ luminescence as a function of vacuum degree.

Figure 5. Distribution of biaxial compressive residual stress in TGO for different oxidation times.

Figure 6. (a) $3 \mathrm{D}$ microstructure of the APS TBCs detected by the X-ray computed tomography method. (b) Pores in top coat marked in dark blue. (c) Dense YSZ region marked in yellow. (d) TGO at interface labeled in black. 
Figure 7. CT slice images showing the microstructure of APS TBCs as a function of thermal oxidation time: (a) 20, (b) 40, (c) 80, (d) 120, (e) 160, and (f) $180 \mathrm{~h}$.

Figure 8. Synchronous measurements of TGO residual stress, TGO thickness, and critical micro-crack length as a function of oxidation time. 
Figures

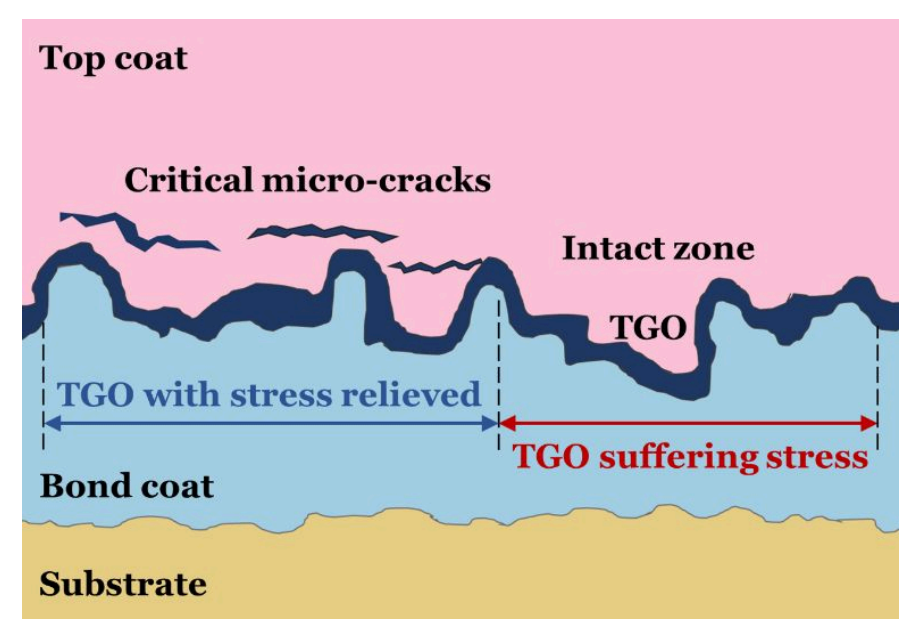

Fig. 1 Schematics of the intact zone at which TGO suffers residual stress and the cracked interface at which TGO stress is relieved.

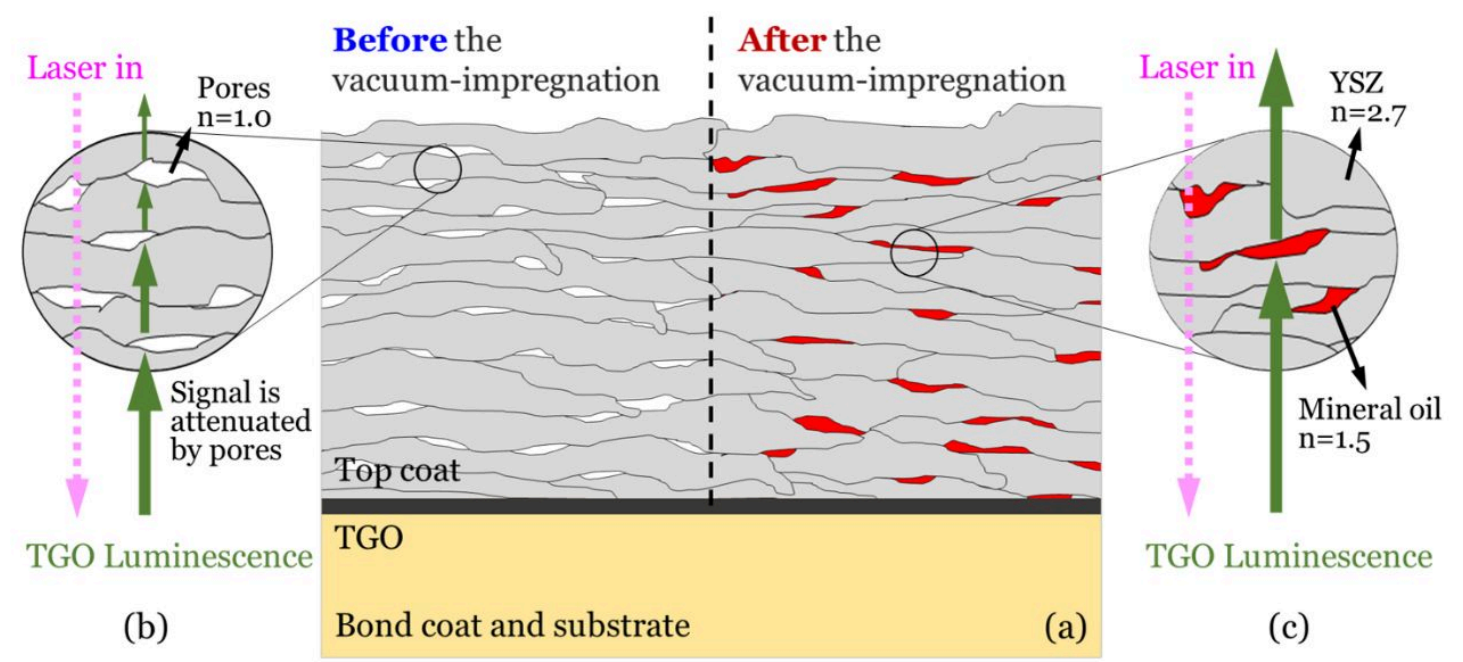

Fig. 2 Schematics showing (a) coating with pores (b) luminescence attenuated by porous ceramic coating. (c) Luminescence penetrating the ceramic coating vacuum impregnated with mineral oil. 


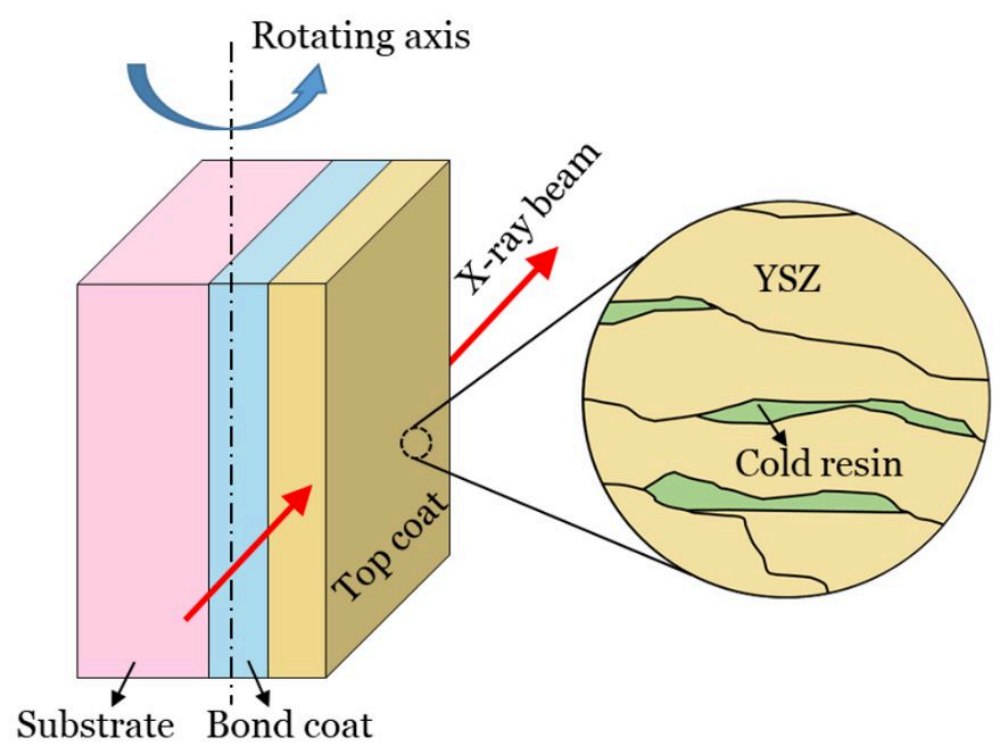

Fig. 3 Schematic describing the X-ray computed tomography test on the TBC sample, which was embedded in a cold mounting resin.

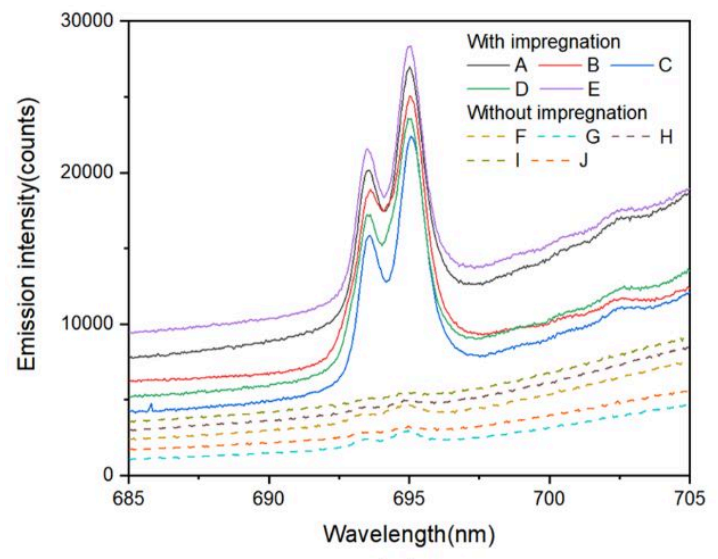

(a)

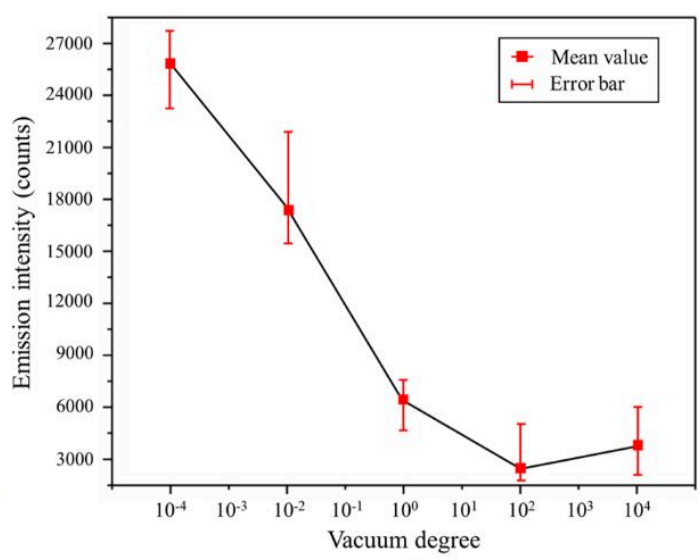

(b)

Fig. 4 (a) Comparison of the detected intensities of $\mathrm{Cr}^{3+}$ luminescence emitted by the TGO and passing through the YSZ TC, before and after the mineral-oil-impregnation treatment, at a vacuum degree of $10^{-4}$. (b) Change in average intensities of $\mathrm{Cr}^{3+}$ luminescence as a function of vacuum degree. 


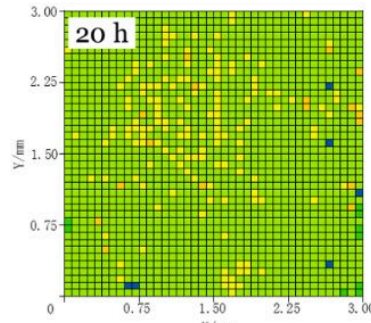

(a)

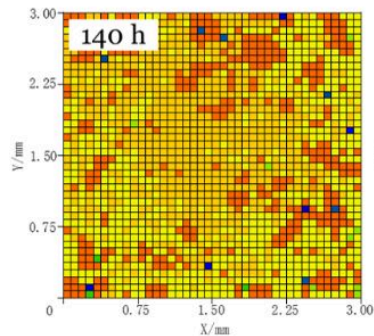

(e)

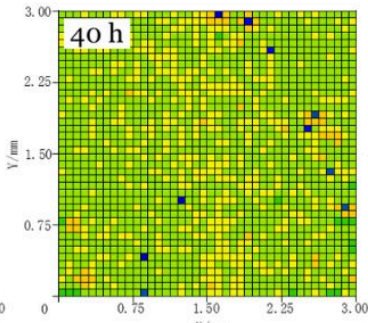

(b)

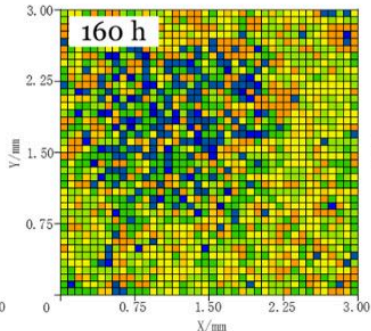

(f)

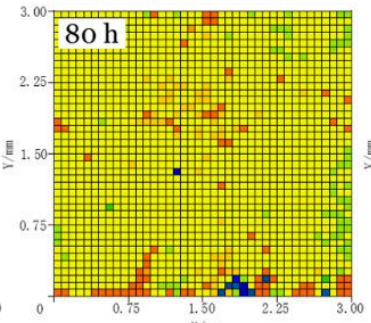

(c)

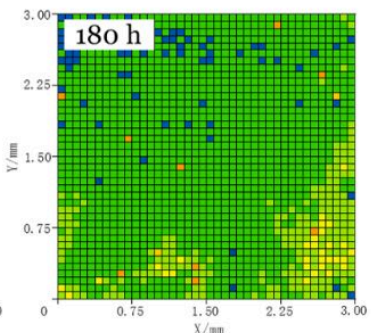

(g)

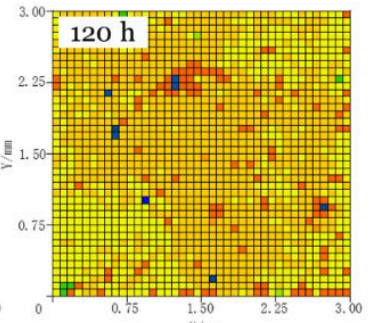

(d)

Compressive residual stress/GPa

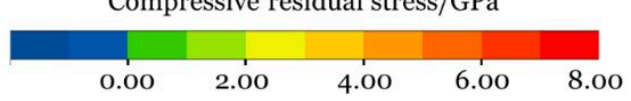

Fig. 5 Distribution of biaxial compressive residual stress in TGO for different oxidation times.
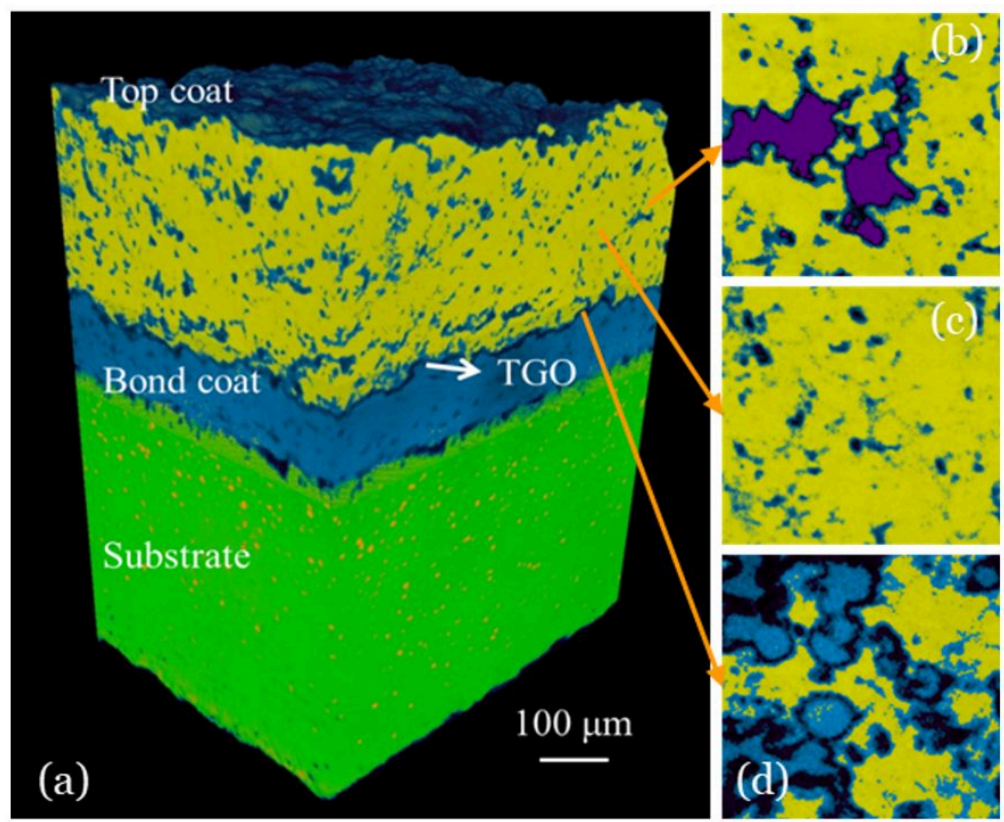

Fig. 6 (a) 3D microstructure of the APS TBCs detected by the X-ray computed tomography method. (b) Pores in top coat marked in dark blue. (c) Dense YSZ region marked in yellow. (d) TGO at interface labeled in black. 

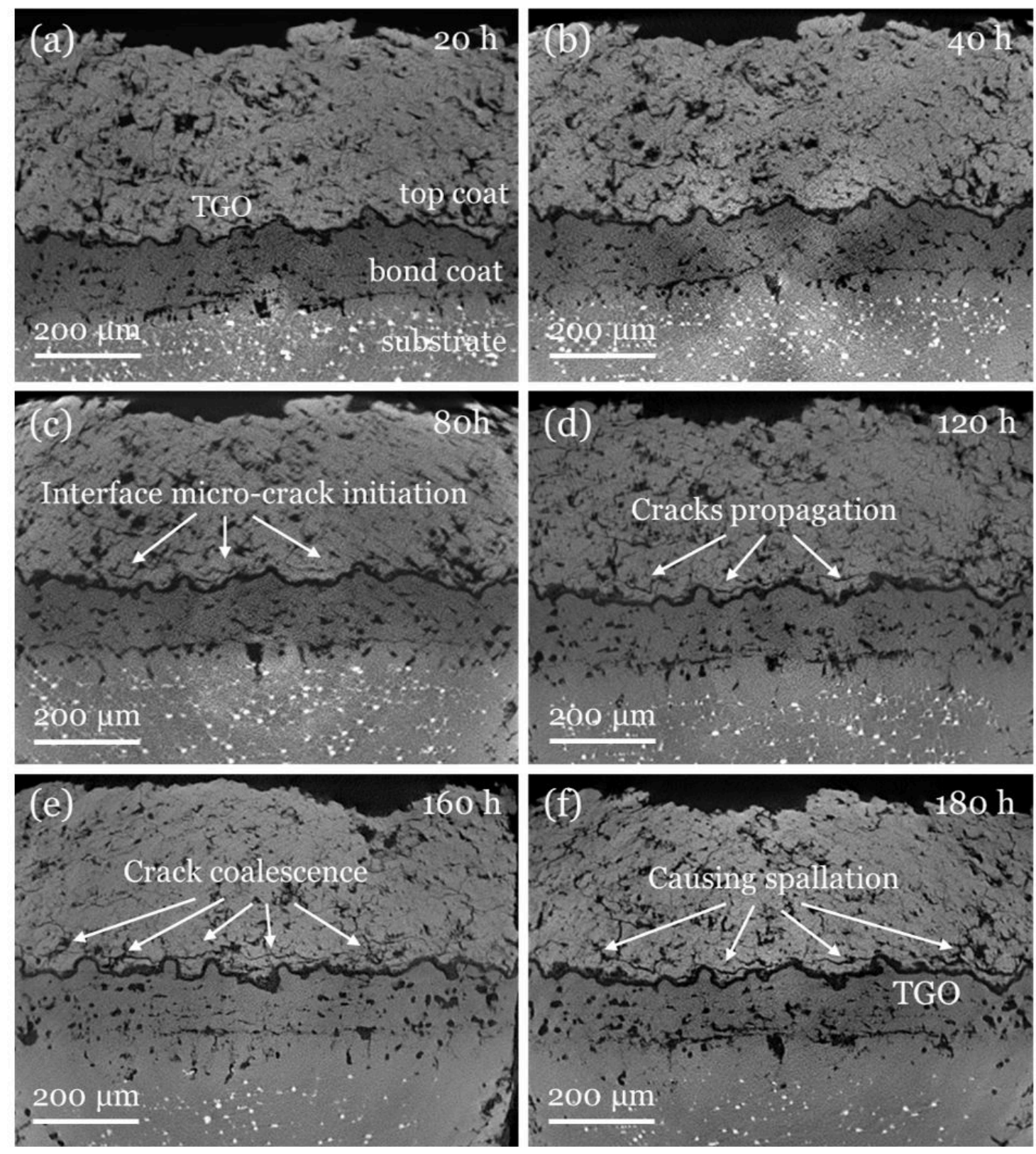

Fig. 7 CT slice images showing the microstructure of APS TBCs as a function of thermal oxidation time: (a) 20, (b) 40, (c) 80, (d) 120, (e) 160, and (f) $180 \mathrm{~h}$. 


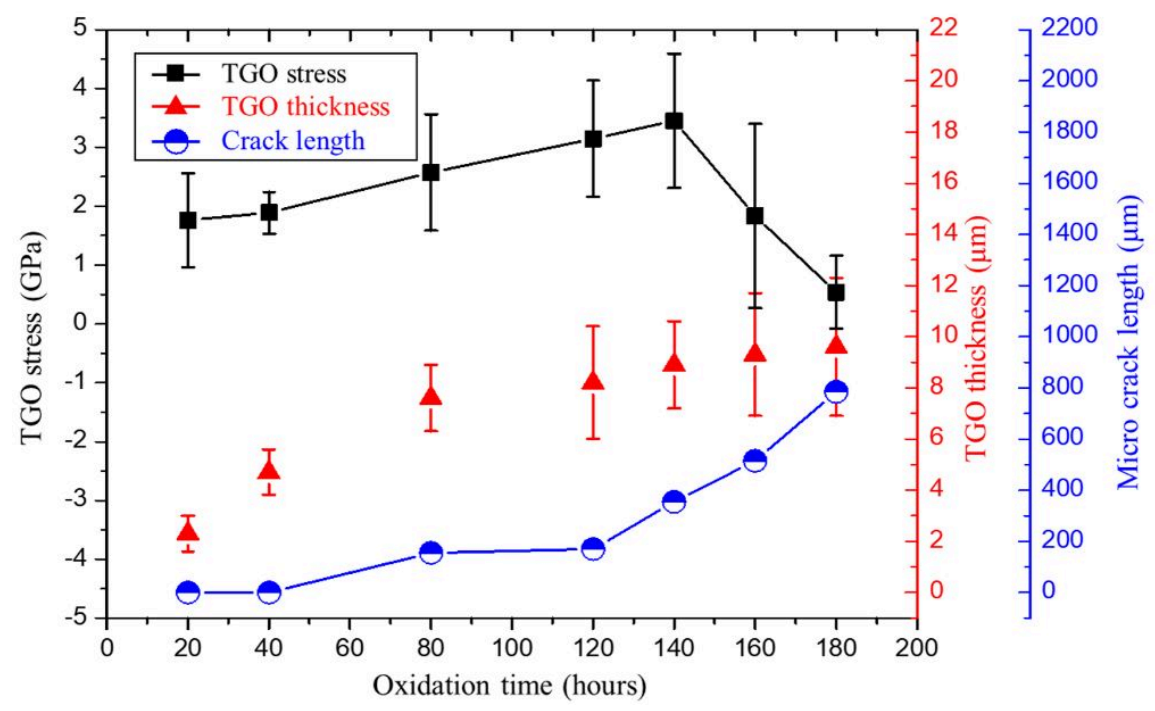

Fig. 8 Synchronous measurements of TGO residual stress, TGO thickness, and critical microcrack length as a function of oxidation time. 
$2020-10-30$

Nondestructive measurements of pÿresidual stress in air plasma sprayed thermal barrier coatings

\author{
Jiang, Peng
}

Wiley

Jiang P, Yang L, Sun Y, et al., (2021) Nondestructive measurements of residual stress in air pÿplasma sprayed thermal barrier coatings. Journal of the American Ceramic Society, Volume 104, Issue 3, March 2021, pp. 1455-1464

https://doi.org/10.1111/jace.17550

Downloaded from Cranfield Library Services E-Repository 